\title{
AVALIAÇÃO SENSORIAL DE DOCE DE LEITE PASTOSO COM DIFERENTES CONCENTRAÇÕES DE AMIDO'
}

\author{
Francisco Eneias KONKEL², Sílvia Maria Rodrigues de OLIVEIRA², Deise Rosana Silva SIMÕES²,
}

\author{
Ivo Mottin DEMIATE ${ }^{3, *}$
}

\section{RESUMO}

O doce de leite é um importante alimento regional, produzido e consumido em grande escala no Brasil e na Argentina. Há grande heterogeneidade da qualidade desse produto, ocorrendo inclusive fraudes com excesso de adição de amido. O presente trabalho teve como objetivo avaliar a preferência de amostras de doce de leite obtidas em laboratório com quantidades conhecidas de amido e a atitude de consumidores frente a duas amostras comerciais, contendo 1,0 e 7,6\% de amido. O método de ordenação foi utilizado na avaliação da preferência das amostras produzidas em laboratório. Verificou-se o nível de aceitação das amostras através de escala hedônica de 9 pontos, no qual 50 julgadores avaliaram as amostras pelos atributos cor, consistência, sabor e doçura. As amostras comerciais foram avaliadas através de uma escala de ação. Nas amostras produzidas em laboratório verificou-se que não houve diferença significativa quanto à preferência $(p>0,05)$ no teste de ordenação e, na avaliação da aceitação por atributos verificou-se que amostras menos doces e com valores intermediários de cor e consistência foram preferidas. Para as amostras comerciais a média de aceitação foi de 6,7 para o produto com teor permitido de amido e 4,9 para aquela com excesso de amido.

Palavras-chave: amido; doce de leite; análise sensorial.

\section{SUMMARY}

SENSORY EVALUATION OF DOCE DE LEITE WITH DIFFERENT CONCENTRATIONS OF STARCH. The doce de leite is an important regional food produced and consumed in large scale in Brazil and Argentina. There are many problems related to the quality standardization, mainly the excess of starch addition. In the present paper the sensory evaluation of samples produced in the laboratory containing known amounts of starch and two commercial samples with 1.0 and 7.6\% of starch was performed. The ranking test was employed for preference evaluation of the laboratory samples. An acceptance test using an hedonic scale was carried out with 50 panelists that evaluated the samples by considering their color, consistency, taste and sweetness. The commercial samples were evaluated through an action scale. For the samples produced in the laboratory there was no significant difference for preference $(p>0.05)$ in the ranking test and for the preference by attributes it was possible to conclude that the less sweet samples and with intermediary values of color and consistency were preferred. The commercial sample containing $1.0 \%$ of starch had an average acceptance of 6.7 whereas the other sample that contained $7.6 \%$ of starch had 4.9 .

Keywords: starch; doce de leite; sensory evaluation.

\section{1 - INTRODUÇÃO}

O doce de leite é um importante alimento produzido e comercializado principalmente na Argentina e no Brasil [15, 21, 23, 26]. É definido como o produto obtido a partir do cozimento de leite adicionado de sacarose, sendo permitido o uso de muitos outros ingredientes [2]. Ainda conforme a legislação, o doce de leite pastoso deve conter teores máximos de umidade de $30 \%$ e de cinzas de $2 \%(\mathrm{p} / \mathrm{p})$. O teor mínimo de proteínas deve ser de $5,0 \%(\mathrm{p} / \mathrm{p})$ e o conteúdo de gordura deve estar entre 6,0 e $9,0 \%$ (p/p). A sacarose é empregada na obtenção do produto e a quantidade máxima admitida no fabrico é de $30 \mathrm{~kg} / 100 \mathrm{~L}$ de leite. Amidos nativos ou modificados são admitidos em proporção não superior a 0,5g/100mL de leite, assim como mono ou dissacarídeos que substituam a sacarose em no máximo $40 \%$ (p/p). A enzima beta-galactosidase (lactase) e o bicarbonato de sódio, podem ser empregados como coadjuvantes, na

\footnotetext{
1. Recebido para publicação em 29/08/2002. Aceito para publicação em 30/01/2004 (000966).

2. Engenharia de Alimentos da UEPG, PIBIC/UEPG/CNPq. E-mail: konkel@bol.com.br

3. Engenharia de Alimentos da Universidade Estadual de Ponta Grossa (UEPG). Av. General Carlos Cavalcanti, 4748 (Campus Uvaranas) CEP: 84030-900. Ponta Grossa, PR Brasil. E-mail: demiate@interponta.com.br * A quem a correspondência deve ser enviada.
}

quantidade necessária para a boa prática de fabricação.

A análise sensorial é um campo muito importante na indústria de alimentos, pois contribui direta ou indiretamente para inúmeras atividades, como desenvolvimento de novos produtos, controle de qualidade, reformulação e redução de custos de produtos, relações entre condições de processo, ingredientes, aspectos analíticos e sensoriais. No teste sensorial é muito importante a padronização das amostras. Muitas vezes o atributo que se pretende avaliar é influenciado por outros fatores, como a quantidade de amostra e a cor do produto [19].

O teste de ordenação é usado quando o objetivo é comparar várias amostras em relação a um simples atributo (como doçura, frescor) ou para avaliar a preferência; neste caso, duas ou mais amostras codificadas são apresentadas simultaneamente. Os julgadores são solicitados a ordená-las de acordo com sua preferência. Como os julgadores são não treinados, não se deve apresentar mais de quatro/cinco amostras para serem ordenadas $[3,16]$.

Os testes afetivos são utilizados quando se necessita conhecer o "status afetivo" dos consumidores com relação ao(s) produto(s), e para isso são utilizadas escalas hedônicas. Dos valores relativos de aceitabilidade se pode inferir a preferência, ou seja, as amostras mais aceitas são as mais preferidas e vice-versa [10]. 
PAULETTI et al. [20] estudaram a relação entre as medidas instrumentais e sensoriais da cor e da textura de nove amostras de doce de leite comerciais, cujas características cobrem o intervalo de variação normal destes atributos no mercado. A cor, a consistência, o tipo de corte e a extensibilidade foram avaliados sensorialmente por uma equipe de provadores treinados, com escalas semiestruturadas. As variações de "luminosidade" (lightness) L* explicaram 92\% da variabilidade de cor detectada sensorialmente.

HOUGH et al. [12] estudaram a taxa de desenvolvimento de cor em sistemas parecidos com o do doce de leite, considerando o teor de sólidos totais, a concentração de bicarbonato de sódio, as proporções de açúcar e as temperaturas de cozimento. Quanto maior a concentração de sólidos, mais intensa a coloração. A maior concentração de bicarbonato de sódio promoveu intenso escurecimento do produto. Quando a sacarose foi parcialmente substituída por frutose ou por glucose, houve maior taxa de desenvolvimento de cor.

ROVEDO, VIOLLAZ \& SUAREZ [24] avaliaram o efeito do $\mathrm{pH}$ e da temperatura no comportamento reológico de amostras de doce de leite comercial argentino. Os autores destacaram a existência de dois tipos de doces de leite na Argentina: o doce de leite para uso doméstico e aquele de uso em confeitaria. O primeiro é consumido como sobremesa e o segundo é preferencialmente usado em confeitaria devido a sua maior viscosidade, que resulta da adição de amido em sua formulação. A composição das amostras analisadas por ROVEDO, VIOLLAZ E SUAREZ [24] era, para aquela de uso doméstico, leite $(80 \%)$, sacarose (16\%) e xarope de glucose (4\%); o doce de leite para confeitaria foi produzido a partir da mesma formulação, adicionado de $2 \%$ de amido, como espessante. Em ambos os tipos de produtos, o conteúdo total de sólidos foi de $71 \%$. Os autores concluíram que a viscosidade aparente do doce de leite de uso doméstico foi alterada pelo $\mathrm{pH}(3,0,4,7,8,3$ e 9,4) e temperatura $\left(25,40\right.$ e $\left.55^{\circ} \mathrm{C}\right)$ das amostras. A acidificação diminuiu e a alcalinização aumentou a viscosidade aparente. Em ambos os casos a viscosidade aparente aumentou durante a armazenagem. Em função do amido adicionado $(2 \%)$, o doce de leite para confeitaria apresentou maior consistência e comportamento mais pseudoplástico que aquele de uso doméstico.

A produção de doce de leite no Brasil é feita por muitas empresas, desde as caseiras até as grandes, com distribuição em todo o país. O doce de leite não apresenta uniformidade de qualidade, apesar de ser produzido em grande volume e amplamente empregado como um ingrediente alimentício, o que pode ser comparado com o que ocorre com o polvilho azedo, outro produto regional $[5,8,9]$.

DEMIATE, KONKEL \& PEDROSO [6] concluíram que de 42 amostras comerciais de doce de leite analisadas, 15 apresentaram excesso de amido, sendo um indicativo de fraude e prejuízo ao consumidor devido à redução do valor nutricional do produto.
O objetivo do presente trabalho foi avaliar o efeito do uso de amido na preferência de amostras de doce de leite pastoso.

\section{2 - MATERIAL E MÉTODOS}

\section{1 - Material}

Seis amostras de doce de leite foram produzidas em laboratório, com diferentes concentrações de amido de milho ácido-modificado (Candymil ${ }^{\circledR}$, Corn Products Brasil, Balsa Nova-PR). Duas amostras foram adquiridas no comércio local de Ponta Grossa-PR, contendo teores conhecidos de amido. Enzimas amilolíticas Termamyl 120L ${ }^{\circledR}$ e AMG300L ${ }^{\circledR}$ (Novozymes, AraucáriaPR) foram empregadas na determinação de amido.

\section{2 - Métodos}

\subsection{1 - Preparo das amostras}

As amostras foram preparadas e numeradas de acordo com a ordem em que foram obtidas, em 1, 2, 3, 4, 5 e 6. A quantidade dos ingredientes utilizados é apresentada na Tabela 1 .

TABELA 1. Guantidade dos ingredientes nas amostras

\begin{tabular}{|c|c|c|c|c|c|c|}
\hline $\begin{array}{ll}\text { Amostra } \\
\text { Ingrediente }\end{array}$ & 1 & 2 & 3 & 4 & 5 & 6 \\
\hline Leite (L) & & & & 3 & & \\
\hline $\begin{array}{l}\text { Xarope a base de } \\
\text { glicose }(g)\end{array}$ & & & & 30 & & \\
\hline Bicarbonato de Sódio(g) & & & & 2,4 & & \\
\hline Açúcar $(g)$ * & & 780 & & 900 & 1090 & 1115 \\
\hline Amido (\%) & 0 & 1,3 & 2,9 & 0,4 & 1,3 & 2,9 \\
\hline
\end{tabular}

* 60\% adicionado no leite e $40 \%$ após caramelização

A quantidade de açúcar nas amostras 4, 5 e 6 foi alterada em relação às amostras 1,2 e 3 , na tentativa de padronizar o teor de açúcares totais e a cor do produto final.

O caramelo foi responsável pelo desenvolvimento da cor característica do doce de leite. A adição de caramelo foi uma adequação do processo tradicional de fabricação, em função das pequenas quantidades de amostras a serem obtidas.

Todas as porcentagens foram calculadas sobre a massa total dos ingredientes.

$\mathrm{Na}$ amostra 4, que deveria conter a mesma quantidade de amido da amostra 1, optou-se por utilizar a quantidade de amido próxima à máxima permitida pela legislação $(0,5 \mathrm{~g} / 100 \mathrm{~mL}$ de leite), uma vez que no mercado predominam os produtos contendo amido [6].

As concentrações de amido foram estimadas para um rendimento de $38 \%$ para as amostras 1 e $4,42 \%$ para as amostras 2 e 5 e $48 \%$ para as amostras 3 e 6 . Dessa maneira, a amostra 1 ficaria com $0 \%$, a amostra 
4 com 1\%, as amostras 2 e 5 com $3 \%$ e as amostras 3 e 6 com $6 \%$ de amido, quando estivessem prontas.

\subsection{2 - Avaliação físico-química}

As amostras foram analisadas com relação à composição química, determinando-se os teores de umidade, açúcares (sacarose, lactose, frutose e glucose), proteínas, lipídios, amido e cinzas.

A umidade foi determinada após secagem em estufa a $105^{\circ} \mathrm{C}$ por 4 horas.

O teor de amido foi analisado após hidrólise enzimática e a glucose quantificada pelo método colorimétrico de glucose oxidase $[4,6,7]$.

Na determinação do teor de sacarose usou-se invertase de Saccharomyces cerevisiae para hidrólise total e procedeu-se a quantificação dos açúcares redutores [18, 27, 29]. Como açúcares redutores, foram detectados lactose, glucose e frutose, simultaneamente [6].

O teor de lipídios foi determinado de acordo com o método de Roese-Gottlieb [14].

As proteínas foram quantificadas pelo método de Micro-Kjeldahl [13].

O teor de cinzas foi determinado após calcinação das amostras em mufla a $550^{\circ} \mathrm{C}$ por 6 horas [13].

\subsection{3 - Avaliação sensorial}

Os testes foram realizados com estudantes universitários e funcionários públicos de ambos os sexos e com idades entre 17 e 50 anos. As amostras foram servidas aleatoriamente aos julgadores em copos plásticos, com quantidades padronizadas $(25 \mathrm{~g}$ - em função do tipo de amostra), e codificadas com 3 dígitos, obtidos de uma tabela de números aleatórios [22]. Foi fornecido biscoito "água e sal" e água para limpeza do palato entre a avaliação das amostras [10]. O teste foi realizado entre 10:00 e 11:00h no laboratório de análise sensorial da Universidade Estadual de Ponta Grossa - UEPG, que dispõe de cabines individuais.

Para avaliar a preferência das amostras 1, 2 e 3 foi utilizado o método de ordenação, solicitando-se aos 100 julgadores que ordenassem as amostras de acordo com a preferência [1].

Nas amostras 4, 5 e 6 para verificar a aceitação pelos atributos cor, consistência, sabor e doçura foi aplicada a 50 julgadores uma escala hedônica de 9 pontos, cujos extremos correspondem a desgostei muitíssimo (1) e gostei muitíssimo (9). As amostras foram apresentadas aos julgadores e foi solicitado que as analisassem com relação a escala proposta. Os resultados referentes às amostras 1, 2, 3 e 4, 5, 6 foram tratados estatisticamente pelo método de Friedman e pela análise de variância empregando-se o software STATISTICA versão 5.0 (StatSoft, Tulsa OK). O delineamento experimental utilizado foi o de blocos completos inteiramente casualizados [10, 25].

Foi também verificada a atitude dos consumidores diante de duas amostras comerciais (A e B) com valo- res extremos de amido ( 1 e 7,6\%) por meio de uma escala de ação para 50 julgadores. A apresentação das amostras foi feita separadamente, solicitando-se aos julgadores que avaliassem a amostra segundo uma escala numérica verbal, que apresenta os seguintes extremos: (1) eu comeria isso se fosse forçado e (9) eu comeria isso em cada oportunidade que tivesse. Os resultados são calculados a partir da média das notas obtidas, e transformados em porcentagem [3, 17, 28].

\section{3 - RESULTADOS E DISCUSSÃO}

\section{1 - Avaliação físico-química}

As massas inicial e final das amostras produzidas em laboratório, determinadas durante o processo de obtenção, permitiram a quantificação, através de cálculos, de todos os constituintes do doce de leite.

Pelas determinações analíticas, foi comprovada a composição calculada e os resultados são mostrados na Tabela 2.

TABELA 2. Composição das amostras produzidas (\%, base úmida)

\begin{tabular}{cccccccccc}
\hline Amostra & Umidade & Glucose & Lactose & Frutose & Sacarose & Lipídios & Proteínas & Cinzas & Amido \\
\hline 1 & 21,0 & 3,8 & 9,6 & 3,0 & 48,0 & 6,5 & 5,7 & 1,5 & 0,0 \\
2 & 29,1 & 3,6 & 8,9 & 2,7 & 38,8 & 5,5 & 4,7 & 1,3 & 2,7 \\
3 & 39,9 & 2,9 & 6,5 & 2,6 & 31,9 & 4,5 & 4,4 & 1,0 & 5,3 \\
4 & 28,8 & 3,4 & 8,3 & 3,1 & 44,0 & 5,1 & 4,8 & 1,1 & 0,9 \\
5 & 27,6 & 3,3 & 7,2 & 2,8 & 45,1 & 4,7 & 4,5 & 1,3 & 2,6 \\
6 & 29,5 & 3,2 & 6,5 & 2,9 & 43,7 & 3,9 & 3,7 & 1,0 & 5,2 \\
\hline
\end{tabular}

De acordo com os resultados mostrados na Tabela 2, o amido determinado apresentou concentrações mais baixas que as esperadas, em função da diferença entre o rendimento esperado e o rendimento obtido. A amostra 3 apresentou excesso de umidade, além do excesso de amido, indicando que o amido adicionado permitiu a obtenção de um "ponto" (consistência) adequado, mesmo com elevado teor de umidade. $\mathrm{Na}$ determinação de açúcares redutores no caramelo, obteve-se uma concentração de $25 \%$ desses açúcares (13\% de glucose), ocorrendo variações de acordo com o grau de caramelização. A sacarose comercial utilizada pode ser considerada de alto grau de pureza (>99\%). Em processos brandos de caramelização da sacarose, ocorre formação de frutose e glucose em quantidades proporcionais, justificando a diferença entre a porcentagem de glucose e os açúcares redutores solúveis determinados no caramelo [11]. Alguns componentes do doce de leite não são mostrados na Tabela 2. Entre eles estão as dextrinas, provenientes do xarope a base de glucose e outros componentes formados na reação de caramelização. Ainda conforme os resultados mostrados na Tabela 2, as amostras 1, 2 e 3 apresentaram variação no conteúdo de sacarose e em conseqüência disso, foram obtidos produtos finais com colorações distintas.

O preparo de novas amostras (4, 5 e 6) resultou em colorações intensas, com pouca variação. A quantidade final de sacarose nas três amostras foi mantida em torno de $44 \%$, o que permitiu a redução na porcenta- 
gem de umidade, de 39,9\%, na amostra 3 para 29,5\%, na amostra 6 (Tabela 2).

\section{2 - Avaliação sensorial}

Os resultados da avaliação sensorial no teste de ordenação das amostras 1, 2 e 3 são mostrados na Tabela 3 e Figura 1.

TABELA 3. Avaliação sensorial de preferência de doce de leite por ordenação para as amostras 1,2 e 3

\begin{tabular}{cc}
\hline Diferença da soma de ordens & Módulos da diferença \\
\hline $1^{\mathrm{a}}-2^{\mathrm{a}}$ & $26(\mathrm{~ns})$ \\
$1^{\mathrm{a}}-3^{\mathrm{a}}$ & $16(\mathrm{~ns})$ \\
$2^{\mathrm{a}}-3^{\mathrm{a}}$ & $10(\mathrm{~ns})$ \\
\hline
\end{tabular}

(ns) = não significativo. Diferença crítica de $\alpha=0,05=34$.

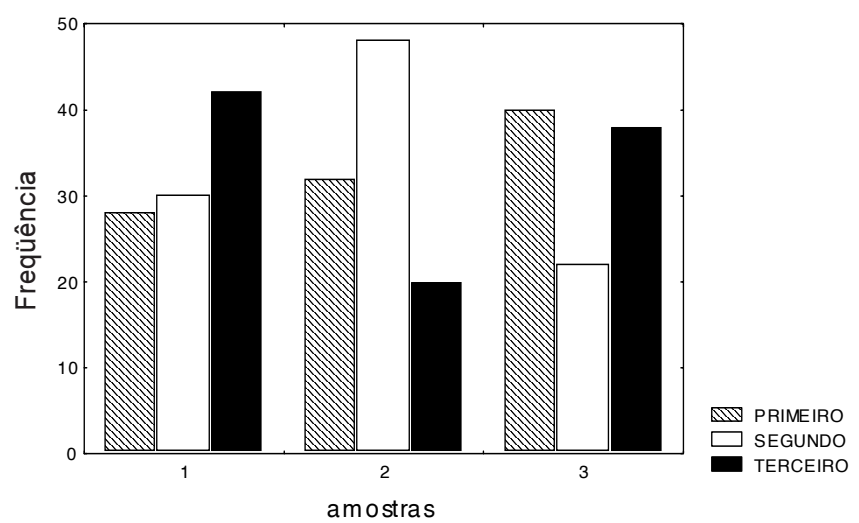

FIGURA 1. Freqüência das ordens das amostras 1,2 e 3

Comparando-se os módulos da diferença com a DMS (diferença mínima significativa), conforme mostrado na Tabela 3, pode-se dizer que não houve diferença estatística significativa entre as amostras ao nível de 5\% de significância.

Observando-se os resultados mostrados na Figura 1, a amostra 3 ficou na primeira posição o maior número de vezes, porém, a melhor média foi observada na amostra 2 , que obteve a maior freqüência na segunda posição e poucas vezes foi escolhida como a pior amostra.

Os resultados da avaliação sensorial no teste de ordenação das amostras 4,5 e 6 são mostrados na Tabela 4 e Figura 2.

TABELA 4. Avaliação sensorial de preferência de doce de leite por ordenação para as amostras 4, 5 e 6

\begin{tabular}{cc}
\hline Diferença da soma de ordens & Módulos da diferença \\
\hline $1^{\mathrm{a}}-2^{\mathrm{a}}$ & $16(\mathrm{~ns})$ \\
$1^{\mathrm{a}}-3^{\mathrm{a}}$ & $23(\mathrm{~ns})$ \\
$2^{\mathrm{a}}-3^{\mathrm{a}}$ & $7(\mathrm{~ns})$ \\
\hline
\end{tabular}

(ns) $=$ não significativo. Diferença crítica de $\alpha=0,05=24$.

Comparando-se os módulos da diferença com a DMS (diferença mínima significativa) pode-se dizer que não houve diferença estatística significativa entre as amostras ao nível de 5\% de significância.

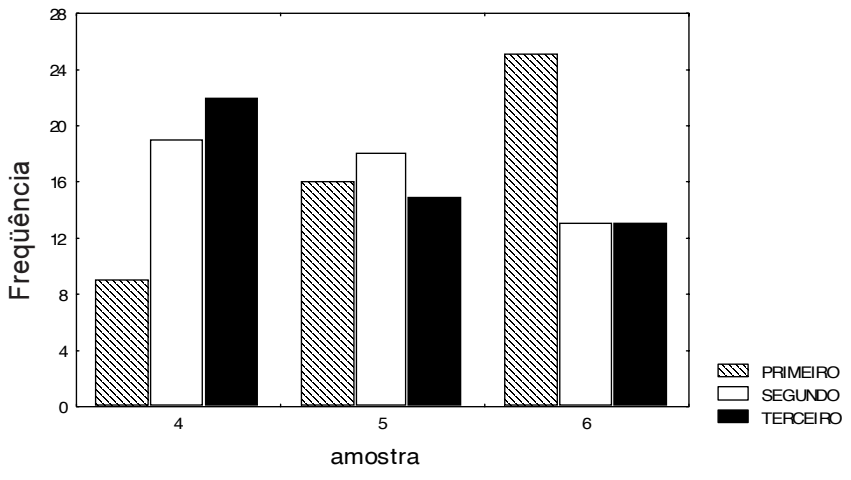

FIGURA 2. Freqüência das ordens das amostras 4, 5 e 6

De acordo com os resultados mostrados na Figura 2, verifica-se que a amostra 6 ficou em primeiro lugar o maior número de vezes. Apesar das amostras 4, 5 e 6 apresentarem concentrações finais de sacarose próximas, o que não aconteceu para as amostras 1, 2 e 3, o resultado do método confirmou não haver diferença significativa entre a preferência pelas amostras.

A Figura 3 representa os resultados obtidos para a avaliação da aceitação para cor, a Figura 4 para consistência, a Figura 5 para sabor e a Figura 6 para doçura das amostras 4,5 e 6 .

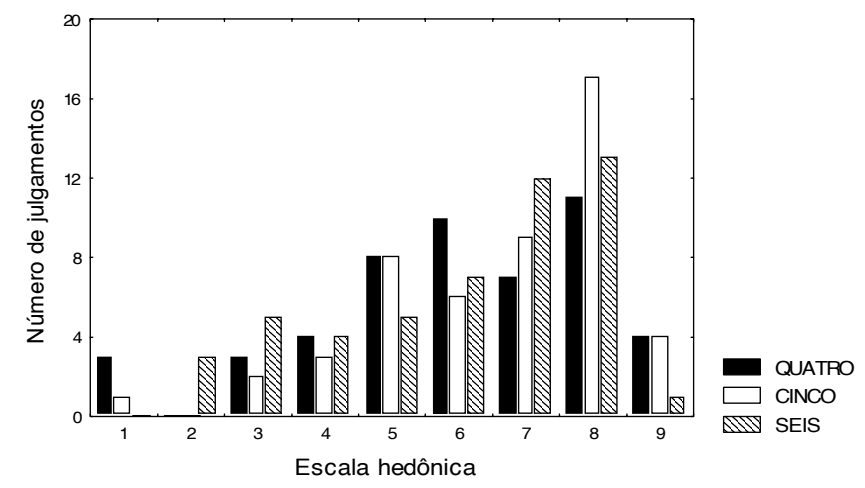

FIGURA 3. Freqüência das notas da escala hedônica para cor das amostras 4,5 e 6

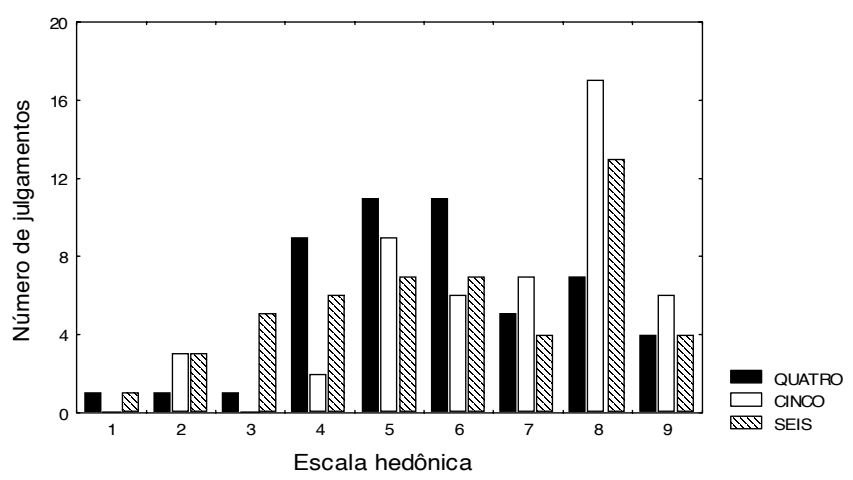

FIGURA 4. Freqüência das notas da escala hedônica para o atributo consistência das amostras 4,5 e 6

Como os maiores valores da escala hedônica equivalem a gostar da amostra, pode-se notar que em relação à avaliação da aceitação pelo atributo cor, sabor e 
consistência a amostra 5 foi a que obteve maior freqüência do índice hedônico (8), que se refere na escala a "gostei muito", e para avaliação pela doçura houve um destaque maior para a amostra 6 , pelo seu maior número de julgamentos referente também a nota 8 , que representa os maiores valores na escala hedônica neste atributo.

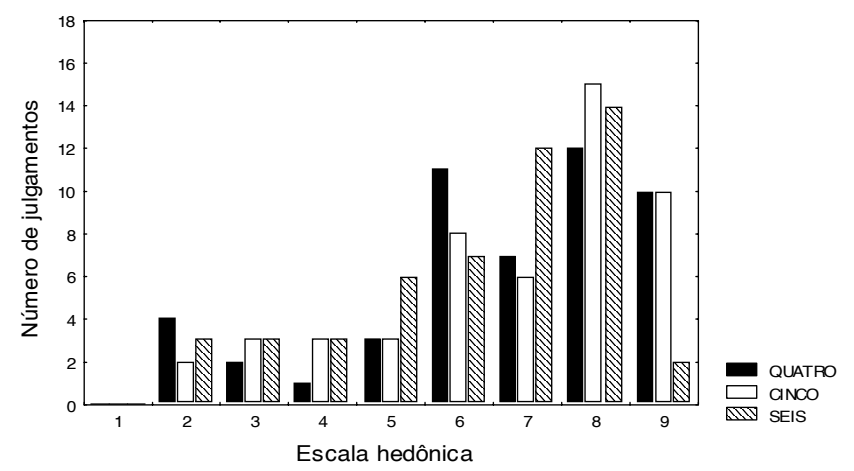

FIGURA 5. Freqüência das notas da escala hedônica para sabor das amostras 4, 5 e 6

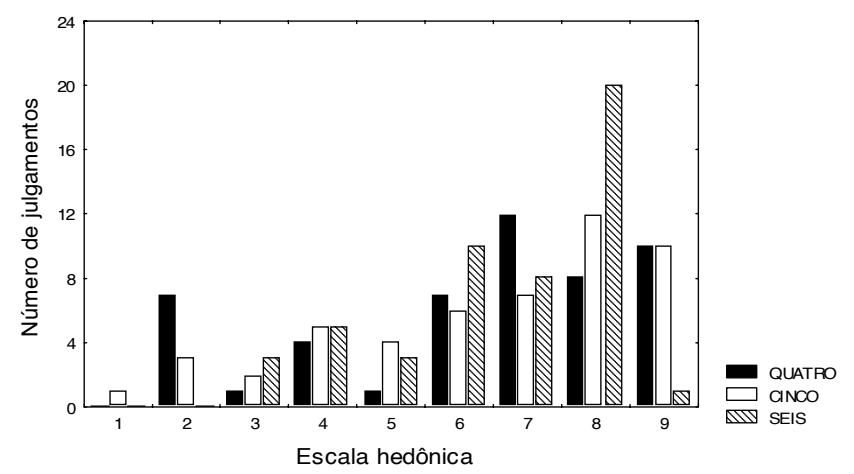

FIGURA 6. Frequência das notas da escala hedônica para doçura das amostras 4,5 e 6

Através da análise de variância, pode-se constatar que os atributos cor $(p=0,27)$, consistência $(p=0,06)$, sabor $(p=0,34)$ e doçura $(p=0,94)$ não apresentaram diferença significativa de aceitação $(p>0,05)$. Estes resultados indicam que a amostra 6 apresentou maior destaque no atributo doçura, mesmo com quantidades de açúcares próximas das amostras 4 e 5 (Tabela 2). Isso pode ser explicado pelo efeito espessante do amido, que diminui a capacidade de dissolução da amostra e de sensibilização das células gustativas. O sabor, apesar de estar intimamente relacionado com a doçura, não apresentou o mesmo resultado de aceitação, o que pode ser explicado pelo fato de que o amido, em excesso, confere ao produto sabor residual indesejado.

Os resultados podem ser também avaliados pela Tabela 5, que apresenta médias de aceitação das amostras 4, 5 e 6 em relação aos atributos cor, consistência, sabor e doçura. Observa-se que as notas atribuídas às amostras (com exceção da consistência) foram superiores a 6, o que representa na escala hedônica um valor considerável.
TABELA 5. Valores médios obtidos a partir da escala hedônica para as amostras 4,5 e 6

\begin{tabular}{ccccccccc}
\hline Amostra & Cor & Erro Padrão Consistência & Ero Padrão & Sabor & Ero Padrão & Doçura & Erro Padrão \\
\hline 4 & 6,0 & 0,3 & 5,8 & 0,3 & 6,7 & 0,3 & 6,4 & 0,3 \\
5 & 6,6 & 0,2 & 6,7 & 0,3 & 6,8 & 0,3 & 6,4 & 0,3 \\
6 & 6,0 & 0,3 & 5,8 & 0,3 & 6,3 & 0,3 & 6,6 & 0,2 \\
\hline
\end{tabular}

As amostras comerciais, codificadas aleatoriamente, contendo valores extremos de amido de 1 e 7,6\%, obtiveram médias de aceitação de 6,7 e 4,9, indicando um grau de aceitabilidade de 74 e 54\%, respectivamente. A Figura 7 mostra a freqüência das notas das amostras A e B.

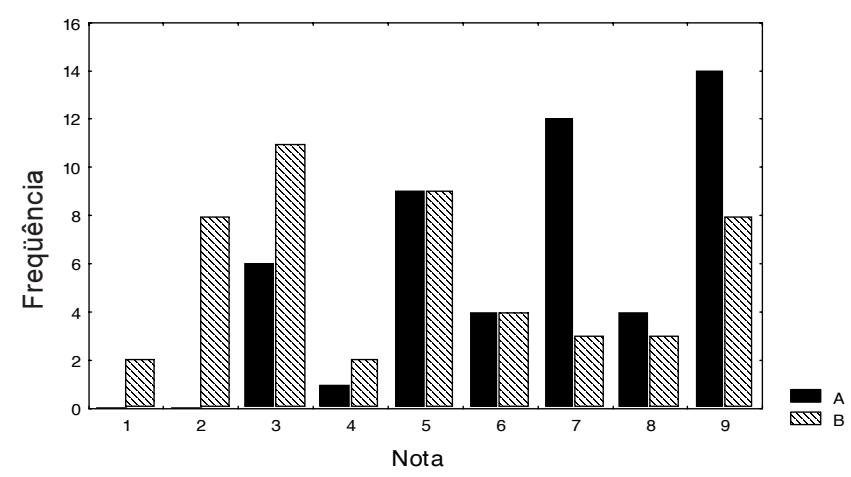

FIGURA 7. Freqüência das notas das amostras A e B, com teores de amido de 1 e 7,6\%, respectivamente

Conforme os resultados mostrados na Figura 7, a amostra A, com 1\% de amido e de melhor qualidade em termos de composição química, obteve uma média de aceitação maior.

\section{4 - CONCLUSÕES}

O preparo de amostras em laboratório permitiu a verificação do efeito da adição de amido, em quantidades acima das permitidas pela legislação, na preferência do doce de leite pastoso.

Pelo teste de Friedman, ao nível de significância de $5 \%$, verificou-se que não houve diferença entre as amostras, o que pode estar associado ao grande consumo de amostras fraudadas encontradas no comércio brasileiro.

Os atributos cor, consistência, sabor e doçura das amostras 4, 5 e 6, não apresentaram diferença significativa ( $>0,05)$, pela análise de variância.

As médias de aceitação para os produtos comerciais com 1 e 7,6\% de amido foram 6,7 e 4,9, respectivamente.

Os resultados mostraram também que os doces de leite comercias com excesso de amido apresentam menor média de aceitação que os doces com quantidades de amido dentro dos limites legais.

\section{5 - REFERÊNCIAS BIBLIOGRÁFICAS}

[1] ABNT. Associação Brasileira de Normas Técnicas. Teste de Ordenação em Análise Sensorial, NBR 13.170, 1994. $7 \mathrm{p}$. 
[2] BRASIL. Ministério da Agricultura e do Abastecimento, Secretaria de Defesa Agropecuária, Departamento de Inspeção de Produtos de Origem Animal. Portaria № 354, de 4 de setembro de 1997. Disponível em: http:// www.agricultura.gov.br/das/dipoa/port354.html Acesso em: 16 nov. 2000.

[3] CHAVES, J.B.P.; SPROESSER, R.L. Práticas de laboratório de análise sensorial de alimentos e bebidas. Universidade Federal de Viçosa: Imprensa Universitária, 1993. 81p.

[4] DAHLQUIST, A. Determination of maltase and isomaltase activities with a glucose oxidase reagent. Biochem.J., v. 80, p. 547-551, 1961.

[5] DEMIATE, I.M.; BARANA, A.C.; CEREDA, M.P.; WOSIACKI, G. Organic acid profile of commercial cassava sour starch. Ciênc. Tecnol. Aliment, v.19, n.1, p.131-135, 1999.

[6] DeMiATE, I.M.; KONKEL, F.E., PEDROSO, R.A. Avaliação da qualidade de amostras comerciais de doce de leite pastoso - composição química. Ciênc. Tecnol. Aliment., v. 21, n. 1, p. 108-114, $2001 \mathrm{a}$.

[7] Demiate, I.M.; KONKEL, F.E., PEDRoso, R.A. Enzymatic determination of starch in doce de leite using dialysis. Ciênc. Tecnol. Aliment., v. 21, n. 3, p. 339342, 2001b.

[8] DEMIATE I.M.; SENGER, S.A.; VOGLER, Z.; CEREDA, M.P.; WOSIACKI, G. Características de qualidade de amostras de polvilho azedo produzido ou comercializado no Estado do Paraná. Arq. Biol. Tecnol, v. 40, n. 2, p. 331-349, 1997a.

[9] DEMIATE, I.M.; SOUZA, T.O. de; PUGSLEY, S.; CEREDA, M.P.; WOSIACKI, G. Características de qualidade de amostras de polvilho azedo. Parte 2. Santa Catarina. Agropec. Catarinense, v. 10, n. 4, p. 51-56, 1997b.

[10]FERREIRA, V.L.P. (Coord.). Análise sensorial - Testes discriminativos e afetivos. Campinas: Sociedade Brasileira de Ciência e Tecnologia de Alimentos, p. 7377, 2000. (Manual Série Qualidade)

[11]Food Science 605. Caramelization, Disponivel em: http:/ /class.fst.ohio-state.edu/FST605/lectures/lect15.html Acesso em: 25 de julho de 2002.

[12] HOUGH, G.; BUERA, M.P.; MARTINEZ, E.; RESNIK, S. Effect of composition on non-enzymatic browning rate in dulce de leche-like systems. Anales de la Associación Química Argentina, v. 79, n. 1, p. 3140, 1991.

[13] IAL. Normas Analíticas do Instituto Adolfo Lutz. Métodos Químicos e Físicos para Análise de Alimentos. $3^{\text {a }}$ ed. São Paulo: IAL, 1985. 533p.

[14] LANARA. Métodos Analíticos Oficiais para Controle de Produtos de Origem Animal e seus Ingredientes. II Métodos físico-químicos. Brasília: Ministério da Agricultura/SNDA/LANARA. Item XV1ll, parte 2, 1981.

[15] MARTINS, J.F.P.; LOPES, C.N. Doce de leite: aspectos da tecnologia de fabricação (Intruções Técnicas, $\mathrm{n}^{\circ}$ 18). Campinas: ITAL, 1980. 37p.
[16] MeIlGAARD, M.; CIVILle, G.V.; CARR, B.T. Sensory evaluation techniques. CRC Press, v. 3, p. 52-292, 1999.

[17] MIÑOZA-GATCHALIAN. Sensory Evaluation Methods with Statistical Analysis. Quezon: University of the Philippines, 1981. 418p.

[18] NELSON, H. A photometric adaptation of the Somogyi method for determination of glucose. Journal of Biological Chemistry, v. 153, p. 375-80, 1944.

[19] PAL, D.; SACHDEVA, S.; SINGH, S. Methods for determination of sensory quality of foods: A critical appraisal. J Food Sci, v. 32, n. 5, p. 357- 367, 1985.

[20] PAULETTI, M.; CALVO, C.; IZQUIERDO, L.; COSTELL, E. Color and texture of Dulce de leche, a confectionary dairy product - Selection of instrumental methods for industrial quality control. Revista Española de Ciência y Tecnologia de Alimentos, v.32, n.3, p.291-305, 1992.

[21] PAYLOVIC, S.; SANTOS, R.C.; SILVA, M.E.; GLORIA, M.B.A. Effect of processing on the nutritive value of Doce de leite, a typical Latin-American confectionary product. Arquivos de Biologia e Tecnologia, v. 35, n. 4, p. 691-698, 1992.

[22]PEDRERO, F.D.L.; PANGBORN, R.M. Evaluación Sensorial de los Alimentos - Métodos Analíticos. Alhambra Mexicana: 103 - 107, 1989.

[23] PINTO, R.V. Doce de leite - Fabricação tradicional. Revista do ILCT, v. 34, n. 205, p. 37-38, 1979.

[24] ROVEDO, C.O.; VIOLLAZ, P.E.; SUAREZ, C. The effect of $\mathrm{pH}$ and temperature on the rheological behavior of Dulce de leche, a typical dairy Argentine product. Journal of Dairy Science, v. 74, n. 5, p. 1497-1502, 1991.

[25] SHIROSE, I.; MORI, E.E.M. Estatística Aplicada a Análise Sensorial. Campinas: ITAL, 1994. 97p. (Manual Técnico n. 13).

[26] SOUZA, G.; OLIVEIRA, A.J.; SHIROSE, I.; VALLE, J.L.E.; CARVALHO, C.R.L. Utilização de abóbora e moranga na produção de doce de leite pastoso com coco. An.ESALQ, v. 47, n. 2, p. 609-623, 1990.

[27] SOMOGYI, M. Determination of blood sugar. Journal of Biological Chemistry, v. 160, p. 69-73, 1945.

[28] TEIXEIRA, E.; MEINERT, E.M.; BARBETTA, P.A. Análise sensorial de alimentos. Florianópolis: Editora da UFSC, 1987. 180p.

[29] VITOLO, M.; CUNHA, B.C.A. Extração de invertase a partir de levedura de panificação: efeito da pressão osmótica e da natureza da solução salina. Rev. Farm. Bioquím. Univ. S. Paulo, v. 17, n. 1, p. 86-92, 1981.

\section{6 - AGRADECIMENTOS}

Os autores agradecem ao CNPq pela concessão de bolsa de iniciação científica e às empresas Corn Products Brasil e Novozymes pelo fornecimento de amido modificado e de enzimas, respectivamente. 